\title{
La internacionalización: implicaciones y modelos
}

\author{
Nelson Eduardo Cottiz Montoya ${ }^{\mathrm{a}}$, Cedrid Gómez Torregrosa ${ }^{\mathrm{b}}$ \& Moisés Alexander Navia Contreras ${ }^{\mathrm{a}}$ \\ ${ }^{a}$ Facultad de Ingeniería, Fundación Universitaria Tecnológico Comfenalco, Cartagena, Colombia. \\ ncottiz@tecnocomfenalco.edu.co, mnavia@tecnocomfenalco.edu.co \\ ${ }^{b}$ Decanatura Académica, Escuela Naval de Cadetes "Almirante Padilla”, Cartagena, Colombia. \\ cedrid.gomez@enap.edu.co
}

Recibido: Septiembre 11, 2019.

Recibido en su versión corregida: Octubre 17, 2019.

Aceptación: Octubre 31, 2019.

Cómo citar: Cottiz Montoya, N.E., Gomez Torregrosa, C. \& Navia Contreras, M.A. (2019). La internacionalización: implicaciones y modelos. Revista Sextante, 20, pp. 44 - 54, 2019.

\section{Resumen}

En la búsqueda de aumentar los ingresos y de captar más clientes para conseguir un mayor beneficio económico, las compañías buscan expandirse a mercados internacionales. A este proceso de expansión se le conoce como internacionalización. Este fenómeno presenta varias formas o modos de entrada de las compañías al mercado internacional, entre las cuales tenemos las exportaciones, acuerdos contractuales, alianzas estratégicas e inversión extranjera directa. Para el ingreso de las compañías a mercados extranjeros se han desarrollado modelos o estructuras que explican este proceso, entre los más reconocidos y estudiados tenemos el modelo Uppsala, también conocido como gradual, los nacidos globales o born glogal y la teoría de redes. En cuanto a la medición de la internacionalización se han definido tres indicadores, estos son: el tiempo, la escala y el alcance.

Palabras claves: Internacionalización, modos de entrada, modelo uppsala, modelo born global, teoría de redes.

\section{Internationalization: implications and models}

\begin{abstract}
In the search to increase revenues and attract more clients to achieve a greater economic benefit, companies seek to expand into international markets. This process of expansion is known as internationalization. Internationalization presents several forms or modes of entry of companies to the international market, among which we have exports, contractual agreements, strategic alliances and direct foreign investment. For the entry of companies into foreign markets, models or structures have been developed that explain this process, among the most recognized and studied we have the Uppsala model, also known as gradual, the global born or born glogal and the network theory. Regarding the measurement of internationalization, three indicators have been defined, these are time, scale and scope.
\end{abstract}

Keywords: Internationalization, entry modes, uppsala model, born global model, network theory. 


\section{Introducción}

La globalización es considerada como un fenómeno que generó opciones para el desarrollo de riqueza para los paises en vías de desarrollo, brindando nuevas oportunidades para la comercialización de servicios y productos de manera global (Javalgi y White, 2002). Este fenómeno ha provocado en países como: India y China, crecimientos económicos altos y estables en las últimas dos décadas. (Khanna, 2009).

El crecimiento de una economía se refleja en el funcionamiento y desempeño de las empresas. En Colombia según Acopi (2017), el tejido empresarial está formado por la gran empresa, con una participación del $1,1 \%$; y la Mipyme, conformada por las microempresas, con una participación del $81,2 \%$; la pequeña empresa, con el 7,5\%; y la mediana empresa, con el 1,5\%. Las Mipymes en Colombia son el gran motor de la economía, dado que generan el $80 \%$ del empleo y aportan el $35 \%$ al producto interno bruto. (Revista Dinero, 2017).

Ahora bien, estudios realizados por el Fondo Monetario Internacional (2017) concluyeron que países como: China, Brasil, Rusia, Turquía, entre otros, tuvieron altos crecimientos debido a la expansión de las exportaciones. Esto nos lleva a pensar que las exportaciones son un medio que promueve el bienestar económico en un país y que genera un impacto positivo al producto interno bruto.

En vista de la importancia de las exportaciones en la salud económica de un país, y teniendo en cuenta que este proceso es uno de varios modos de entrada al mercado internacional, la internacionalización cobra gran interés, porque en ella se describen otras formas de ingreso de la compañía al exterior, identifica formas y modelos que describen este proceso, y además, como lo advierte (Felício, Duarte y Rodrigues, 2016), la internacionalización es crucial para las micro, pequeñas y medianas empresas en su desarrollo, especialmente si estas provienen de pequeños países.

\section{Metodología}

Se realizó una búsqueda de artículos relacionados con la internacionalización, sus modelos y las implicaciones en las economías.

En este apartado se encuentran los fundamentos metodológicos, discriminando las acciones a realizar en cada fase del proyecto.

La metodología que se plantea corresponde al paradigma de interpretación también llamado cualitativa. Situación que permite establecer una estrecha relación entre este paradigma y el método propuesto por Hoyos (2000), donde se evidencian la investigación de tipo descriptivo, que corresponde a los estados del arte. Entonces, los planteamientos metodológicos de esta propuesta se desarrollan en cinco fases, que son:

\section{Desarrollo y discusión}

\section{La internacionalización.}

El proceso de expansión de una empresa, más allá de su dominio interno, se le conoce como internacionalización (Alonso et al., 2013). Para Welsh y Loustarinen (1993) este fenómeno es un movimiento al exterior de las operaciones internacionales de la empresa. Por lo anterior observamos que la internacionalización es el proceso en el cual una empresa mueve desde su lugar de origen, hacia el exterior sus procesos, productos y servicios, pero para realizar este movimiento, como lo advierte Beamish (1990), esta tiene que determinar, a través de actos empresariales, qué implica, buscar y detectar oportunidades internacionales que tengan el potencial de influir en el futuro de la organización, y que además establezcan y conduzcan transacciones en otros países. Welch y Luostarinen (1988) definen la internacionalización como el proceso de incrementar la inversión en operaciones internacionales; por su parte Calof y Beamish (1995) la definen como el proceso de adaptar las operaciones de la empresa como son la estrategia, recursos y estructura al contexto internacional. Para McDougall y Oviatt (2000) la internacionalización es un proceso donde se asume una combinación de comportamientos innovativos, proactivos y de riesgo que cruzan las fronteras buscando crear valor en las organizaciones. Para Czinkota y Ronkainen 
(2007) la base de la internacionalización son las relaciones empresariales o personales que brinden ventajas para el ingreso al mercado, así mismo Johanson y Vahle (2009) señalan que la internacionalización es un proceso de desarrollo de redes de negocios en otros países, a través de la extensión, penetración e integración.

La internacionalización es un proceso que trae consigo múltiples beneficios, entre los cuales tenemos: la mejora de la competitividad, reflejando un crecimiento significativo de la inversión extranjera directa y de los flujos comerciales (Botello, 2016); promueve el crecimiento de la compañía y el desarrollo de la estrategia de la misma (Agnieszka y Anetta, 2015); tiene un impacto positivo en el rendimiento de la empresa (Delios y Beamish, 2001); permite establecer contactos con nuevos clientes, generando un conocimiento especializado del mercado (Soriano y Dobon, 2009); es un proceso que permite atraer el interés de clientes de diferentes países (Lane y Jacobson, 1995); promueve oportunidades para que la empresa logre economías de alcance y de escala. (Pangarkar, 2008)

Ahora bien, para medir el proceso de internacionalización Kuivalainen et al (2007) propone tres dimensiones: el tiempo, la escala y el alcance. El tiempo para Autio et al (2000) es una dimensión importante para analizar, dado que determina la estructura o modelo de internacionalización de la empresa. Para Zucchella et al (2007) esta dimensión obedece a la longitud del tiempo entre el inicio de la compañía y su primera internacionalización. Otra dimensión es la escala, la cual para Kuivalainen et al (2007) abarca el alcance de las operaciones internacionales de la empresa, y el indicador usado para medir esta dimensión según Baum, Schwens y Kabst (2015) y Geringer, Tallman y Olsen (2000) es el porcentaje de las ventas extranjeras sobre las ventas totales. En cuanto al alcance, como lo señala Tuppura et al (2008), se centra en la extensión de la internacionalización, y su indicador más aplicado, como lo advierte Zahra et al (2000), es el número de mercados de países extranjeros donde una empresa tiene actividades internacionales.

\section{Factores que promueven la internacionalización}

En el proceso de internacionalización en las empresas, se han definido y resaltado ciertos factores que la promueven, entre estos tenemos: el mercado, la experiencia, los recursos, la diversificación del riesgo, la competencia, el cambio tecnológico, las redes y el aumento de los ingresos.

En cuanto al mercado, Rialp et al. (2005) advierte que un emprendedor, quien ha desarrollado un producto o servicio puede obtener más utilidades ingresando a mercados similares al primero. Esto obedece claramente a que la empresa, por medio del conocimiento obtenido y a la información recopilada en su aventura internacional, se convierte en un elemento de gran utilidad para poder usarse en el ingreso a nuevos mercados internacionales. Por lo tanto, como lo afirma Yeoh (2004), el conocimiento del mercado y la habilidad para asimilar la información son componentes importantes de un proceso de internacionalización empresarial. Otra condición que motiva el inicio del proceso de internacionalización es el tamaño del mercado local o doméstico (Murmann, Ozdemir y Sardana, 2015), dado que, si el mercado local es pequeño, la empresa buscará expandirse a uno o más países extranjeros en búsqueda de una mayor demanda (Gertler y Levitte 2005), o también con el fin de apalancar financieramente sus operaciones. (Murmann, Ozdemir y Sardana, 2015)

Un segundo factor que incide en el inicio de la internacionalización empresarial es la experiencia internacional previa, la cual le permite a la empresa mejorar en el proceso de identificación de oportunidades (Westhead et al 2001), en las elecciones de los modos de entrada (Anderson y Gatignon, 1986) y en el grado de la internacionalización (Reuber y Fischer, 1997). Para Aaby y Slater (1989) el tiempo que lleva la empresa exportando tiene una gran influencia en su trayectoria, que se refleja en la experiencia, como también en el know-how obtenido anteriormente, esto le permite a la compañía sobrepasar las dificultades logísticas. De igual forma hay que anotar también el rol que juega la experiencia de los directivos y equipos en las empresas ante la internacionalización, ya que como lo señala Reuber $\&$ Fischer (1997) y Siegel (2004) la experiencia previa que tienen los quipos directivos influye de 
manera positiva en el proceso de internacionalización.

Toda empresa que desee incursionar en mercados internacionales necesitará disponer cierta cantidad de recursos para lograr su objetivo. Ante esto, Sui y Baum (2014) advierten que los recursos son un factor determinante en las estrategias de internacionalización en las empresas, y en vista de su importancia y del impacto que tiene en este proceso, las empresas pueden adquirir de una manera más fácil capital financiero en países extranjeros, debido a la internacionalización de los mercados financieros. (Murmann, Ozdemir y Sardana, 2015)

Luiz y Charalambous (2009) realizaron un estudio a bancos en el sur de África en torno a la internacionalización, y encontraron que una motivación para expandirse al extranjero es la diversificación del riesgo, por lo tanto, este es un factor que promueve la internacionalización dado que las empresas realizan distribuciones de sus recursos en una gran variedad de proyectos e inversiones con el fin de reducir o mitigar el riesgo.

Otro factor que motiva la internacionalización empresarial como lo advierte McDougall et al. (1994) es la competencia, la cual en este caso se traduce como el temor de los empresarios de que competidores potenciales en otros países puedan rápidamente copiarlos y luego desafiarlos en su país de origen. Según Oviatt y McDougall (1994) el empresario está motivado a proteger sus utilidades en el mercado doméstico, y teniendo en cuenta que la competencia puede imitarlos y con eso introducir los productos al mercado local del empresario, este escenario futuro impulsaría a que el empresario se internacionalice para evitar que las empresas de otros países imiten los productos y construyan capacidades que puedan competir con sus propios productos y capacidades, y con esto, como lo señala 489 , llevar el enfrentamiento al propio país de los competidores, para que no tengan que pelear con ellos en casa.

El cambio tecnológico es otro factor que mejora la habilidad para internacionalizarse (Murmann, Ozdemir y Sardana, 2015). Las innovaciones en este campo han permitido la reducción de los costos de la comunicación, los viajes y el transporte de bienes y servicios. (Rialp et al., 2005) Botello (2016) reconoce que los componentes tecnológicos son facilitadores y activos específicos de la empresa, que juegan un papel diferenciador en la estructura de la empresa y motivan su inmersión al mercado internacional; así mismo Skudiene et al., (2015) destaca la importancia del internet en el proceso de internacionalización cuando manifiesta que, la disponibilidad de información basada en internet es un antecedente importante de éxito en el proceso de internacionalización.

Otro factor que destacamos para el inicio del proceso de internacionalización en las empresas son las redes, las cuales para Rask et al (2008) destacan gran ayuda para que ocurra el proceso de internacionalización. Para Johanson y Mattson (1988) la internacionalización inicia cuando la empresa toma en consideración su entorno de red empresarial, siendo para Welch y Loustarinen (1993) las redes de contactos personales y la interacción social, claves en la entrada al mercado internacional.

Las empresas en la búsqueda de su beneficio económico y financiero que permita aumentar sus ingresos ven la internacionalización como una oportunidad, tal como lo señala Rask et al (2008), el cuál advierte que la compañía busca internacionalizarse para sobrevivir y maximizar sus ingresos.

\section{Modos de entrada al mercado internacional}

La compañía según Cateora, Gilly y Graham (2011) tiene cuatro formas para entrar a un mercado extranjero, estas son: la exportación, acuerdos contractuales, alianzas estratégicas e inversión extranjera directa. Los autores señalan que en cada modo de entrada el capital requerido varía, por lo tanto, según la cantidad de dinero que se use, este afecta el riesgo, el retorno de la inversión y el control.

Basándonos en la Figura 1, tomada de Cateora, Gilly y Graham (2011) y teniendo en cuenta lo descrito anteriormente, podemos identificar que las exportaciones se caracterizan porque la cantidad de capital es bajo, por consiguiente, habrá menos riesgo, baja tasa de retorno y poco control, mientras que el modo de entrada inversión extranjera directa, requiere un mayor capital que los otros modos de 
entrada, por lo que genera un riesgo mayor, mayor control y una alta tasa de retorno.

La exportación para Cateora, Gilly y Graham (2011) puede ser directa o indirecta. La exportación directa es aquella cuando la compañía vende a un cliente en otro país. Y la exportación indirecta ocurre cuando la compañía vende su producto o servicio a un comprador, es decir, a un importador o distribuidor.

Otro modo de entrada al mercado internacional son los acuerdos contractuales, los cuales para Cateora, Gilly y Graham (2011) representan alianzas a largo plazo entre una compañía y otra en un mercado extranjero. Este tipo de acuerdos involucran transferencia de tecnología, procesos o talento humano. En definitiva, este modo de entrada sirve más bien para transferencia de conocimiento que de capital. Entre los tipos de acuerdos los autores resaltan las licencias y las franquicias. El primero es un medio en el cual se establece un apoyo en el mercado extranjero sin el uso de grandes cantidades de dinero. Derechos de patentes, derechos de comercialización y derechos de uso de procesos tecnológicos son ejemplos de licencias. Por su parte, la franquicia, los autores la sintetizan como una licencia que ha tenido un rápido crecimiento, en el cual el franquiciador proporciona un paquete de productos, sistemas y servicios de administración, y el franquiciado provee el conocimiento del mercado, capital y personal.

En cuanto al modo de entrada a alianzas estratégicas internacionales, Cateora, Gilly y Graham (2011) las describen como una relación de negocios establecidos por dos o más compañías con el fin de cooperar de mutuo acuerdo, compartiendo los riesgos, con el fin de lograr un objetivo en común. Entre los más comunes se destacan los join venture internacional y los consorcios. Por último, el modo de entrada a inversión extranjera directa para Cateora, Gilly y Graham (2011) se fundamenta en la inversión de grandes cantidades de capital en un país extranjero, por lo que la compañía puede invertir, comprar locales, o establecer nuevas instalaciones operacionales.

\section{Modelos de internacionalización}

Kłysik-Uryszek y Kuna-Marszałek (2015) apuntan que los cambios en la economía global han impulsado el surgimiento de modelos que describen la expansión internacional de las empresas, tanto así que este proceso es variado, tal como lo afirma Grosman et al, (2005), quien advierte que puede realizarse haciendo exportaciones de manera directa o indirecta, estableciendo una filial de servicios o instalaciones de producción en el mercado exterior, o puede combinar cualquiera de estos modelos.

Los modelos de internacionalización varían significativamente en cuanto a los indicadores y en la aplicación del modelo (Baum, Schwens y Kabst, 2015). Entre los modelos o teorías que explican el proceso de internacionalización tenemos los descritos por Gorynia (2007), que los divide en internacionalización de tipo activa y pasiva. La activa se enmarca en la expansión en mercados extranjeros, en cambio la pasiva se enfoca en la colaboración y establecimiento de vínculos económicos con aliados o socios extranjeros en el país en el cual la compañía en cuestión se basa. Por otro lado, Kłysik-Uryszek y Kuna-Marszałek (2015) describen un modelo de internacionalización que se sustenta en tres etapas, la experimental, la activa y el compromiso de involucrarse en el proceso (committed involvement). La primera etapa (la experimental), se enfoca y se limita a la exportación, el cual es un proceso totalmente improvisado. En la segunda etapa (la activa), las actividades de internacionalización se enmarcan en una estrategia formal en la empresa, en la cual el proceso de exportar se convierte en un proceso sistemático. Y en la última etapa, (committed involvement), la empresa se encuentra en plena búsqueda de oportunidades para internacionalizarse en mercados extranjeros, lo cual le implica a la compañía involucrarse dentro de nuevas formas de internacionalización.

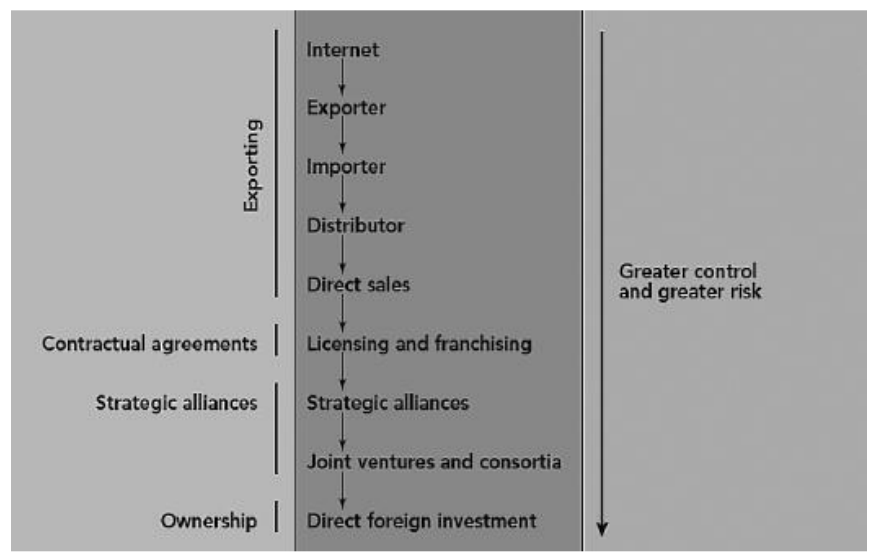

Figura 1. Estrategias para entrar al mercado.

Fuente: Los autores. 
Para Tuppura et al., (2008) los modelos, estructuras o teorías de internacionalización más reconocidos, aceptados y estudiados son el Uppsala, también conocido como gradual, los nacidos globales o born global y la teoría de redes.

\section{Modelo Uppsala}

Este modelo se fundamentó en observaciones empíricas producto de un estudio en el área de negocios internacionales en la universidad Uppsala, realizado por Johanson y Vahlne (1977), las cuales mostraron que empresas suecas cuando entraron a mercados extranjeros adoptaron un enfoque por etapa para su ingreso en el exterior. La internacionalización de estas empresas se dio como resultado de la adquisición gradual, la integración y el aprovechamiento del conocimiento sobre los mercados y las operaciones exteriores y los incrementos progresivos de los compromisos con los mercados extranjeros. En definitiva, el modelo Uppsala se sustenta en el desarrollo, inicialmente en el mercado doméstico, para luego desarrollar su proceso de internacionalización con una serie de pasos incrementales (Skudiene et al., 2015) y en el compromiso de la compañía con el mercado y con el conocimiento de este (Forsgren y Hagstrom, 2007).

Johanson y Vahlne (1990) señalan que el modelo se compone de cuatro etapas. La primera etapa consiste en exportaciones accidentales, en esta fase la compañía no adquiere experiencia del mercado. El siguiente momento es la venta de productos o servicios a través de agentes independientes. Aquí la compañía tiene un canal de información del mercado. La tercera etapa se da cuando la compañía establece una sucursal en el exterior, lo cual le permite adquirir más conocimiento del mercado meta. Y la cuarta y última etapa comprende en establecer sus propias instalaciones en el extranjero. La compañía al momento de recibir conocimiento de otros mercados y del aprendizaje del proceso de internacionalización, puede superar las barreras para su crecimiento y operar de forma más eficiente en mercados internacionales. Johanson y Vahlne (1977) utilizan el concepto "psychic distance" (distancia psíquica) en el modelo Uppsala, para relacionarlo con culturas diferentes o diferentes prácticas de negocios en los países de origen y de acogida. Los autores señalan que para que la empresa minimice el riesgo de operar en el extranjero, esta primero se expande a mercados que son más cercanos en términos de psiquis, es decir, países que tienen una cultura similar y que geográficamente estén cerca al domicilio de la compañía, cuando la compañía adquiere suficiente experiencia, esta le sirve para entrar a mercados donde la distancia psíquica es mayor.

Johanson y Vahlne (2009) advierten sobre la estructura de internacionalización tradicional. La empresa busca minimizar el riesgo y la incertidumbre, y operar en el exterior bajo un esquema de internacionalización de pasos, comprometiéndose por aprender sobre su entorno, lo cual genera un conocimiento que le permitirá a la compañía incrementar el compromiso con el mercado extranjero, teniendo en cuenta, como lo advierte Ruzzier (2006), que el modelo implica que más que invertir en varios países simultáneamente, la compañía deberá tomar pequeños pasos incrementales e invertir dentro de uno al menos o en pocos países cercanos. Kłysik-Uryszek y KunaMarszałek (2015) en su estudio dirigido en la forma en que empresas polacas ingresaron a mercados extranjeros, encontraron que en el proceso de internacionalización iniciaron con exportaciones indirectas, las cuales más tarde se convirtieron en exportaciones directas a graduales, además concluyeron que las empresas iniciaron invirtiendo en el mercado europeo, particularmente en países vecinos y cercanos en geografía y en términos culturales, concluyendo así que las empresas polacas se enmarcaron en un modelo conservativo para internacionalizarse, el cual se ajusta al modelo Uppsala.

Ahora bien, este modelo ha sido reconocido como el más aplicable a diferentes economías y en diferentes compañías (Skudiene et al., 2015), es uno de los más comunes para estudiar la internacionalización (Kłysik-Uryszek y KunaMarszałek (2015) y coinciden que su gran fortaleza es su simplicidad y su importancia. (Fillis, 2001)

\section{Modelo Born Global o Nacidos Globales}

Al inicio de 1990 diversos investigadores de emprendimiento comenzaron a notar que nuevas empresas en formación ofrecían sus productos en varios países (Oviat y McDougall, 1994). Este tipo de empresas, conocidas como born global, están apareciendo en un número importante a nivel 
mundial (Eurofound, 2012), lo cual se debe a la globalización, el internet y otras innovaciones en comunicación. Knight y Cavusgil (2004) definen la empresa born global como un emprendimiento que desde su inicio buscó derivar una proporción substancial de su ingreso de las ventas de productos en mercados internacionales. Para Oviat y McDougall (1994) este tipo de empresas, desde sus inicios, buscan importantes ventajas significativas desde el uso de sus recursos y de las ventas de sus productos en varios países. Las empresas que siguen este modelo según Rialp, Rialp y Knight (2005) se caracterizan por ser bastantes flexibles en sus decisiones y se adaptan fácilmente a las necesidades del cliente y los requerimientos de la competencia. Para Baum, Schwens y Kabst, (2015) la estructura born global es asociada con una gran exposición al mercado internacional a una edad temprana. En definitiva, las empresas que se internacionalicen de manera temprana y que logren ventas en el exterior desde su inicio, son conocidas como las nacidas globales o born global.

Para distinguir si una compañía sigue un modelo born global hay que tener en cuenta ciertos indicadores, como son: la precocidad, la cual para Zucchella et al (2007) es la longitud del tiempo entre el inicio de la compañía y su primera internacionalización; y el porcentaje de las ventas generadas en el extranjero. Ante el primer indicador observamos que no hay un consenso definido, ya que para que una empresa tenga la característica de ser born global, según Knight y Cavusgil (2004), tuvo que haberse internacionalizado dentro los primeros tres años, después de su comienzo. Para Moen y Servais (2002) el tiempo es de dos años y para Fernhaber et al (2007) es de 6 años después de su comienzo. En cuanto al indicador porcentaje de las ventas generadas en el exterior, evidenciamos que no hay un parámetro definido, dado que hay autores como Knight y Cavusgil (2004) que proponen que la empresa tiene que generar el $25 \%$ de sus ventas en el extranjero. Para Fan y Phan (2007) este indicador tiene que ser del $20 \%$, y para Lopez et al (2009) tiene que ser el $90 \%$ de sus ventas en el extranjero.

Las empresas que siguen una estructura o modelo born global según Andersson y Wictor (2003) cuestionan la naturaleza secuencial y acumulativa del modelo de internacionalización tradicional o gradual, y más bien apuntan a la posibilidad de que la empresa tenga opciones simultaneas en muchos mercados extranjeros, es decir, la compañía decide si se compromete en realizar inversiones en mercados extranjeros sin tener experiencia en operaciones económicas en ese país. Por otro lado, Baum, Schwens y Kabst, (2015) señalan que la internacionalización de las empresas born global no se desarrolla de manera lenta e incremental, sino de una manera bastante proactiva. Por su parte Sleuwaegen y Onkelinx (2014) destacan que este tipo de empresas expanden su negocio al exterior saltándose los pasos y llegando a la etapa de exportación, ofreciendo sus productos a muchos mercados a la misma vez, reportando altos incrementos en las ventas como las empresas que siguen el modelo de internacionalización tradicional o gradual.

\section{Modelo de Redes}

Zhong y Ozdemir (2010) advierten que la continua expansión del conocimiento y las dificultades de adquirir y mantener recursos, hacen que las empresas necesiten unir fuerzas con otras organizaciones por la vía de asociaciones formales. Las asociaciones o alianzas, como lo señala Hagedoorn (2002), se han convertido en la piedra angular para la estrategia de la empresa, con el fin de buscar el éxito en el mercado. Las empresas crean asociaciones o redes para complementar sus propias habilidades (Oviatt y McDougall, 1994), para obtener recursos, descubrir oportunidades, reducir el riesgo y la incertidumbre en operaciones internacionales (Liesch et al., 2002), como también para facilitar la adquisición de conocimiento y el desarrollo de recursos (Selnes y Sallis, 2003). Las empresas que buscan internacionalizarse por este modelo de redes, son empresas jóvenes, las cuales por falta de experiencias y recursos realizan alianzas para acceder y desarrollar mercados internacionales (Contractor y Lorange, 1998), también este modelo es aplicado por aquellas empresas de países que presentan demandas locales pequeñas (Murmann, Ozdemir y Sardana, 2015).

En vista de los beneficios de las redes, las empresas se apalancan en ellas para entrar a mercados internacionales. Möller y Rajala (2007) describen que el modelo de redes asume que las empresas adquieren experiencia desde la relación de negocios con otras compañías, operando en una red internacional. Así mismo, señalan que este modelo 
de internacionalización es el resultado de interacciones entre una empresa y la red de relaciones con clientes, competidores, proveedores, consultores y otras entidades del entorno internacional.

\section{Conclusiones y recomendaciones}

La internacionalización es sin duda una herramienta que permite a la compañía expandirse a mercados internacionales, buscando aumentar sus ingresos por medio de la captación y atracción de nuevos clientes, además promueve mejoras en el desempeño de la empresa, haciéndola más competitiva.

En el proceso de internacionalización se identifican tres dimensiones para su medición, estas son: el tiempo, la escala y el alcance. La primera abarca el tiempo medido desde su creación hasta el momento de su primera internacionalización; la segunda se fundamenta en el porcentaje de las ventas realizadas en el exterior sobre las ventas totales; y la última dimensión comprende el número de mercados de países extranjeros donde una compañía tiene operaciones.

Ahora bien, hay factores que promueven e inducen a la empresa a su internacionalización, entre estas tenemos el mercado, la experiencia previa, los recursos, el riesgo, la competencia, la tecnología, las redes y los ingresos.

Entonces, si una empresa está motivada a ingresar al mercado internacional, lo puede hacer de diferentes formas, una de ellas son las exportaciones, las cuales pueden ser de manera directa o indirecta; otra es los acuerdos contractuales, entre los cuales se encuentran las licencias y las franquicias; tenemos también las alianzas estratégicas internacionales, entre estas tenemos a los consorcios y los join venture; y por último la inversión extranjera directa.

La expansión de las empresas a mercados internacionales originó modelos que describen y explican este proceso. Entre los modelos se encuentra el uppsala, el cual es de corte tradicional, donde la empresa se internacionaliza por pasos secuenciales a mercados extranjeros que tienen una cultura similar al domicilio donde se encuentra la compañía. Otro modelo es el born global o nacidos globales, en el cual se enmarcan aquellas compañías que se han expuesto de manera temprana a mercados internacionales logrando ventas en el exterior. Y tenemos también la teoría de redes, en la cual la compañía se internacionaliza apalancándose con otras empresas, desarrollando así una red internacional o apoyándose en las relaciones construidas con los clientes, proveedores, y competidores.

\section{Referencias}

Aaby, N. E. \& Slater, S. F. (1989). Management influences on export performance: A review of the empirical literature 1978-1988. International Marketing Review, 6(4)

Acopi. (2017). Competitividad y reforma tributaria. Boletin

Agnieszka Kłysik-Uryszek, \& Anetta KunaMarszałek. (2015). Geographical breakdown of Polish OFDI -explanatory potential of Uppsala model. Journal of International Studies, Vol. 8, No 1, 2015, pp. 150-163. DOI: 10.14254/2071$8330.2015 / 8-1 / 13$

Alonso, J.M., Clifton, J., Diaz-Fuentes, D., Fernandez-Gutierrez, M., Revuelta, J., 2013. The race for international markets: were privatized telecommunications incumbents more successful than their public counterparts? Int. Rev. Appl. Econ. 27, 215e236.

Anderson, E., \& Gatignon, H. (1986). Modes of foreign entry: A transaction cost analysis and propositions. Journal of International Business Studies, 17: 1-26.

Anderson, E., \& Gatignon, H. (1986). Modes of foreign entry: A transaction cost analysis and propositions. Journal of International Business Studies, 17: 1-26.

Andersson S., Wictor I. (2003), Innovative Internationalisation in New rms: Born Globalsthe Swedish Case, "Journal of International Entrepreneurship”, Vol.1(3), pp. 249-275.

Autio, E., Sapienza, H. J., \& Almeida, J. G. (2000). Effects of age at entry, knowledge intensity, and 
imitability on international growth. Academy of Management Journal, 43(5): 909-924.

Baum, M., Schwens, C., \& Kabst, R. (2015). A latent class analysis of small firms' internationalization patterns. Journal of World Business $\quad 50 \quad$ (2015) 754-768. http://dx.doi.org/10.1016/j.jwb.2015.03.001

Beamish, P. W. (1990). The internationalization process for smaller Ontario firms: A research agenda. In A. M. Rugman (Ed.), Research in global strategic management - International business research for the twenty-first century: Canada's new research agenda (pp. 77-92). Greenwich: JAI Press Inc.

Botello Peñaloza, H.A. (2016). Las certificaciones de calidad y la internacionalización de las firmas industriales colombianas. Suma de Negocios (2016),

http://dx.doi.org/10.1016/j.sumneg.2016.02.009

Calof J., Beamish P.W. (1995), Adapting to foreign markets: explaining internationalization, “International Business Review”, Vol. 4(2), pp. 115-131.

Cateora, P., Gilly, M., \& Graham, M. (2011). International marketing. 15th ed. Published by McGraw-Hill. ISBN 978-0-07-352994-3

Contractor, F.J., Lorange, P. (Eds.), (1988). Lexington Books, Lexington, MA.

Czinkota, M., \& Ronkainen, I. (2007). International marketing (8th ed.). Cincinnati: Cengage.

Delios, A., \& Beamish, P. W. (2001). Survival and profitability: The roles of experience and intangible assets in foreign subsidiary performance. Academy of Management Journal, 44(5): 1028-1038.

Eurofound (2012). Born global: The potential of job creation in new international businesses. Luxembourg: Publications Office of the European Union.

Fan, T., \& Phan, P. (2007). International new ventures: Revisiting the influences behind the 'born-global" firm. Journal of International Business Studies, 38: 1113-1131.

Felício, J., Duarte, M., y Rodrigues, R. (2016). Global mindset and SME internationalization: A fuzzy-set QCA approach. Journal of Business Research $69 \quad$ (2016) 1372-1378. http://dx.doi.org/10.1016/j.jbusres.2015.10.110

Fernhaber, S. A., McDougall, P. P., \& Oviatt, B. M. (2007). Exploring the role of industry structure in new venture internationalization. Entrepreneurship: Theory and Practice, 31: 517-542.

Fondo Monetario Internacional. (2017). Perspectivas de la economía mundial; En busca del crecimiento sostenible: Recuperación a corto plazo, desafios a largo plazo. Washington (octubre). ISSN 1564-5215

Forsgren, M., \& Hagström, P. (2007). Ignorant and impatient internationalization? The Uppsala model and internationalization patterns for Internet-related firms. Critical Perspectives on International Business, 3, 291

Geringer, J. M., Tallman, S. \& Olsen, D. M. (2000). Product and international diversification among Japanese multinational firms. Strategic Management Journal, 21(1), 51-80.

Gorynia M. (2007), Strategie zagranicznej ekspansji przedsiębiorstw, PWE, Warszawa.

Grossman, G., Helpman, E., Szeidl, Á., (2005). Complementarities between outsourcing and foreign sourcing. Amer. Econ. Rev. 95 (2), 1924.

Hagedoorn, J., (2002). Inter-firm $R \& D$ partnerships: an overview of major trends and patterns since 1960. Res. Policy 31 (4), 477-492.

Javalgi, R., \& White, D. (2002). Strategic challenges for marketing of services internationally. International Marketing Review, 19(6): 563-581.

Johanson I., Vahlne I. (1990), e Mechanism of Internationalization, "International Marketing Review”, Vol. 7(4), pp.11-24. 
Johanson J., Vahlne J.E. (1977), e internationalization process of the $\mathrm{rm}$ - A model of knowledge development and increasing market commitments, "Journal of International Business Studies”, Vol. 8(1), pp. 23-32.

Johanson, J. \& Mattsson, L. (1988). Internationalization in industrial systems $-A$ network approach. In Strategies in global competition. Ed. By Hood, N., \& Vahlne, J.-E., pp.287-314. London: Croom Helm

Johanson, J., \& Vahle, J.-E. (2009). The Uppsala internationalization model revisited-form liability of foreignness to liability of outsidership. Journal of International Business Studies, 40, 1411-1431.

Khanna, T. (2009). China and India, the power of two. Harvard Business Review 85(12): 60-69.

Kłysik-Uryszek, Agnieszka., y Kuna-Marszałek, Anetta. (2015). Geographical breakdown of Polish OFDI - explanatory potential of Uppsala model. Journal of International Studies, Vol. 8, No 1, 2015, pp. 150-163. DOI: 10.14254/2071$8330.2015 / 8-1 / 13$

Knight, G., \& Cavusgil, S. T. (2004). Innovation, organizational capabilities, and the born-global firm. Journal of International Business Studies, 35(2): 124-141.

Kuivalainen, O., Sundqvist, S., \& Servais, P. (2007). Firms' degree of born-globalness, international entrepreneurial orientation and export performance. Journal of World Business, 42(3): 253-267.

Lane, V., \& Jacobson, R. (1995). Stock market reactions to brand extension announcement: The effects of brand attitude and familiarity. Journal of Marketing, 59: 63-77.

Liesch, P. W., Welch, L. S., Welch, D., McGaughey, S. L., Petersen, B., \& Lamb, P. (2002). Evolving strands of research on firm internationalization: An Australian-Nordic perspective. International Studies of Management \& Organization. 32. (pp. 16-35).
Lopez, L. E., Kundu, S. K., \& Ciravegna, L. (2009). Born global or born regional? Evidence from an exploratory study in the Costa Rican software industry. Journal of International Business Studies, 40(7): 1228-1238.

Luiz, J.M., Charalambous, H., (2009). Factors influencing foreign direct investment of South African financial services firms in Sub-Saharan Africa. International Business Review 18 (3), 305-317.

McDougall, P. P., \& Oviatt, B. M. (2000). International entrepreneurship: The Intersection of two research paths. Academy of Management Journal, 43(5): 902-908.

Moen, Ø., \& Servais, P. (2002). Born global or gradual global? Examining the export behavior of Small and medium-sized enterprises. Journal of International Marketing, 10(3): 49-72.

Möller K., Rajala A. (2007), Rise of strategic nets New modes of value creation, "Industrial Marketing Management, Vol. 36(7), pp. 895908.

Murmann, J., Ozdemir, S., \& Sardana, D. (2015). The role of home country demand in the internationalization of new ventures. Research $\begin{array}{llll}\text { Policy } & 44 & \text { (2015) 1207-1225. }\end{array}$ http://dx.doi.org/10.1016/j.respol.2015.03.002

Oviatt, B. M., \& McDougall, P. P. (1994). Toward a theory of international new ventures. Journal of International Business Studies, 25(1): 45-64.

Pangarkar, N., (2008). Internationalization and performance of smalland medium sized enterprises. J. World Business 43, 475---485.

Rask, M., Strandskov, J., \& Hakonsson, D.D. (2008). Theoretical Perspectives on the Internationalization of Firms. Journal of Teaching in International Business, 19, 320-345.

Reuber, A. R., \& Fischer, E. (1997). The influence of the management team's international experience on the internationalization behaviors of SMEs. Journal of International Business Studies, 28(4): 807-825. 
Revista Dinero. (2017). Los retos que enfrentan las mypimes en Colombia. Revista Dinero. Recuperado de http://www.dinero.com/edicionimpresa/pymes/articulo/los-retos-queenfrentan-las-mipymes-en-colombia/241586

Rialp, A., Rialp, J., Knight, G.A., (2005). The phenomenon of early internationalizing firms: what do we know after a decade (1993-2003) of scientific inquiry? Int. Bus. Rev. 14 (2), 147-166.

Ruzzier M., Hisrich R.D., Antončič B. (2006), SME internationalization research: past, present, and future, "Journal of Small Business and Enterprise Development”, Vol.13(4), pp. 476497.

Selnes, F., \& Sallis, S. (2003). Promoting relationship learning. Journal of Marketing, 67(3), 80-89.

Siegel, C. (2004). Internet marketing: foundations and applications. Boston: Houghton Miffin.

Skudiene, V., Auruskeviciene, V., \& Sukeviciute, L. (2015). Internationalization model revisited: $e$ marketing approach. 20th International Scientific Conference Economics and Management - 2015 (ICEM-2015). Procedia Social and Behavioral Sciences 213 (2015) 918 -924. doi: 10.1016/j.sbspro.2015.11.505

Sleuwaegen L., Onkelinx J. (2014), International commitment, post-entry growth and survival of international new ventures, "Journal of Business Venturing”, Vol. 29(1), ss. 106-120.

Soriano, D. R., \& Dobon, S. R. (2009). Linking globalization of entrepreneurship in small organizations. Small Business Economics, 32(3), 233-239.

Sui, S., Baum, M., (2014). Internationalization strategy, firm resourcesand the survival of SMEs in the export market. J. Int. BusinessStud. (in press).

Tuppura, A., Saarenketo, S., Puumalainen, K., Jantunen, A., \& Kyla“heiko, K. (2008). Linking knowledge, entry timing and internationalization strategy. International Business Review, 17: 473-487.
Welch L.S., Luostarinen R.K. (1988), Internationalization: Evolution of a Concept, "Journal of General Management", Vol. 14(2), pp. 36-64.

Welsh, L.S., \& Loustarinen, R.K. (1993). Inward and outward connections in internationalization. Journal of International Marketing, 1, 46-58.

Westhead, P., Wright, M., \& Ucbasaran, D. (2001). The internationalization of new and small firms: A resource-based view. Journal of Business Venturing, 16: 333-358.

Yeoh, P. L. (2004). International learning: Antecedents and performance implications among newly internationalizing companies in an exporting context. International Marketing Review, 21(4-5), 511-534.

Zahra, S. A., Ireland, R. D., \& Hitt, M. A. (2000). International expansion by new venture firms: International diversity, mode of market entry, technological learning, and performance. Academy of Management Journal, 43(5): 925950.

Zhong, X., Ozdemir, S.Z., (2010). Structure, learning, and the speed of innovating: a twophase model of collective innovation using agent-based modeling. Ind. Corp. Change 19(5), 1459-1492.

Zucchella, A., Palamara, G., \& Denicolai, S. (2007). The drivers of the early internationalization of the firm. Journal of World Business, 42(3): 268280. 